TRANSACTIONS OF THE

AMERICAN MATHEMATICAL SOCIETY

Volume 178, April 1973

\title{
SUBMANIFOLDS AND A PINCHING PROBLEM ON THE SECOND FUNDAMENTAL TENSORS
}

BY

\section{MASAFUMI OKUMURA}

ABSTRACT. This paper gives a sufficient condition for a submanifold of a Riemannian manifold of nonnegative constant curvature to be totally umbilical. The condition will be given by an inequality which is established between the length of the second fundamental tensor and the mean curvature.

Introduction. In a previous paper [4] the present author proved the following theorem.

Theorem A. Let $M$ be an $n$-dimensional, connected, compact bypersurface with constant mean curvature immersed in an $(n+1)$-dimensional Riemannian manifold of nonnegative constant curvature. If the second fundamental tensor $H$ satisfies

$$
(n-1) \text { trace } H^{2}<(\text { trace } H)^{2},
$$

then $M$ is a totally umbilical bypersurface and consequently a sphere.

In this theorem, if the length of the second fundamental tensor is constant, then we can replace the condition that $M$ is compact by requiring that $M$ is complete. In this case, the estimation in Theorem $A$ is the best possible since $S^{n-1}$ $\times E^{1}$ in $E^{n+1}$ satisfies $(n-1)$ trace $H^{2}=(\text { trace } H)^{2}$.

In this paper, the author tries to generalize this theorem to a submanifold of any codimension which is immersed in a Riemannian manifold of nonnegative constant curvature.

1. Preliminaries. Let $M$ be an $n$-dimensional submanifold of an $(n+p)$-dimensional Riemannian manifold $\bar{M}$ of constant curvature $c$. The Riemannian connections of $M$ and $\bar{M}$ are denoted by $\nabla$ and $\bar{\nabla}$ respectively, whereas the connection in the normal bundle of $M$ in $\bar{M}$ is denoted by $D$. Let $N_{1}, \ldots, N_{p}$ be mutually orthogonal unit normal vectors at a point $P \in M$ and extend them to vector fields in a neighborhood of $P$. We define $-H_{A} X(A=1,2, \cdots, p)$ to be the tangential component of $\bar{\nabla}_{X} N_{A}$ for $X \in T_{p}(M)$ and call $H_{A}$ the second fundamental

Received by the editors April 13, 1972.

AMS (MOS) subject classifications (1970). Primary 53C40, 53C20. pinching.

Key words and phrases. Second fundamental tensor, totally umbilical submanifold, 
tensor with respect to $N_{A}$. Then we have the following equations of Gauss and Weingarten:

$$
\begin{gathered}
\bar{\nabla}_{X} Y=\nabla_{X} Y+\sum_{A=1}^{p} g\left(H_{A} X, Y\right) N_{A}, \\
\bar{\nabla}_{X} N_{A}=-H_{A} X+D_{X} N_{A},
\end{gathered}
$$

where $g$ is the Riemannian metric of $M$. We know that the $H_{A}$ 's are symmetric linear transformations on $T_{p}(M)$. Since the ambient manifold is of constant curvature $c$, the scalar curvature $K$ and the normal curvature $R^{N}$ are respectively given by

$$
K=n(n-1) c+\sum_{A=1}^{p}\left(\operatorname{trace} H_{A}\right)^{2}-\sum_{A=1}^{p} \operatorname{trace} H_{A}^{2}
$$

$$
R^{N}(X, Y) N_{A}=\sum_{B=1}^{p} g\left(\left[H_{A}, H_{B}\right] X, Y\right) N_{B} .
$$

From (1.4) we see that $R^{N}=0$ at $P$ if and only if $H_{A} H_{B}=H_{B} H_{A}$ at $P$ for all $A, B=1,2, \cdots, p$, that is, the $H_{A}$ 's are simultaneously diagonalizable at $P$. Also, $R^{N}=0$ everywhere if and only if for each $P \in M$ we can choose $p$ mutually orthogonal unit normal vector fields $N_{1}, \ldots, N_{p}$ on a neighborhood $U$ of $P$ such that $D N_{A}=0$ in $U$. If $R^{N}=0$ at $P \in M$ we say that the connection of a normal bundle is flat at $P$.

The mean curvature vector $N$ is defined by

$$
N=\sum_{A=1}^{p}\left(\operatorname{trace} H_{A}\right) N_{A},
$$

and it is well known that $N$ is independent of the choice of unit normal vectors to $M$.

If there exist $p$ functions $\rho_{A}(A=1, \cdots, p)$ such that

$$
H_{A} X=\rho_{A} X, \quad A=1, \cdots, p,
$$

at each point of $M$, we call $M$ a totally umbilical submanifold. From (1.6), for a totally umbilical submanifold, we have

$$
\rho_{A}=(1 / n) \text { trace } H_{A} \text {. }
$$

2. Laplacian of the square of the length of the second fundamental tensor. We put

$$
f=\sum_{A=1}^{p} \operatorname{trace} H_{A}^{2}
$$

Then, from (1.3) and (1.5) we know that $f$ is independent of the choice of 
mutually orthogonal unit normal vectors $N_{A}(A=1, \ldots, p)$ to $M$ and thus $f$ is a globally defined function on $M$.

We assume that the mean curvature vector field of the submanifold $M$ is parallel with respect to the connection in the normal bundle, that is, $D N=0$. Then we easily get that

$$
\sum_{A=1}^{p}\left(\text { trace } H_{A}\right)^{2}=\text { constant }
$$

Recently K. Yano and S. Ishihara [6] and J. A. Erbacher [1] have obtained the following formula for the Laplacian of the function $f$ :

$$
\begin{aligned}
1 / 2 \Delta f= & c\left(n f-\sum_{A=1}^{p}\left(\operatorname{trace} H_{A}\right)^{2}\right)+\sum_{A, B=1}^{p} \operatorname{trace}\left[H_{A}, H_{B}\right]^{2} \\
& +\sum_{A, B=1}^{p}\left(\operatorname{trace} H_{A}\right)\left(\text { trace } H_{A} H_{B}^{2}\right)-\sum_{A, B=1}^{p}\left(\text { trace } H_{A} H_{B}\right)^{2} \\
& +\sum_{A=1}^{p} g\left(\nabla^{*} H_{A}, \nabla^{*} H_{A}\right),
\end{aligned}
$$

where we extend the metric $g$ to the tensor space in the standard fashion and $\nabla *$ denotes the connection in the Whitney sum of the tangent bundle of $M$ and the normal bundle of $M$ induced by $\nabla$ and $D$.

Suppose that the connection of the normal bundle is flat. Then by the discussion in $\$ 1,(2.3)$ can be written in the following form:

$$
\begin{aligned}
1 / 2 \Delta f= & c\left(n f-\sum_{A=1}^{p}\left(\operatorname{trace} H_{A}\right)^{2}\right)+\sum_{A, B=1}^{p}\left(\text { trace } H_{A}\right)\left(\text { trace } H_{A} H_{B}^{2}\right) \\
& -\sum_{A, B=1}^{p}\left(\operatorname{trace} H_{A} H_{B}\right)^{2}+\sum_{A=1}^{p} g\left(\nabla^{*} H_{A}, \nabla^{*} H_{A}\right) .
\end{aligned}
$$

Now we state a lemma which can be easily proved by mathematical induction. have

Lemma 2.1. For any set of $2 n$ real numbers $\left\{a_{1}, \ldots, a_{n}, b_{1}, \cdots, b_{n}\right\}$, we

$$
\left(\sum_{i=1}^{n} a_{i}\right)\left(\sum_{j=1}^{n} a_{j} b_{j}^{2}\right)-\left(\sum_{i=1}^{n} a_{i} b_{i}\right)^{2}=\sum_{i<j}^{n} a_{i} a_{j}\left(b_{i}-b_{j}\right)^{2} .
$$

Next we prove

Lemma 2.2. If a given set of $n+1(n \geq 2)$ real numbers $a_{1}, \cdots, a_{n}$ and $k$ satisfies the inequality

$$
\sum_{i=1}^{n} a_{i}^{2}+k^{2}<\frac{1}{n-1}\left(\sum_{i=1}^{n} a_{i}\right)^{2}
$$


then, for any pair of distinct $i$ and $j=1, \ldots, n$, we have

$$
k^{2}<2 a_{i} a_{j}
$$

Proof. Since $\left(\sum_{i=1}^{n} a_{i}\right)^{2}=\sum_{i=1}^{n} a_{i}^{2}+2 \sum_{i<j}^{n} a_{i} a_{j}$, we have from (2.6),

$$
(n-2) \sum_{i=1}^{n} a_{i}^{2}-2 \sum_{i<j}^{n} a_{i} a_{j}+(n-1) k^{2}<0,
$$

that is,

$$
(n-2) a_{n}^{2}-2 a_{n}\left(\sum_{i=1}^{n-1} a_{i}\right)+(n-2) \sum_{i=1}^{n-1} a_{i}^{2}-2 \sum_{i<j \leq n-1} a_{i} a_{j}+(n-1) k^{2}<0 .
$$

We regard that (2.8) is a quadratic inequality with respect to $a_{n}$. Then, $a_{n}$ being a real number, the discriminant of $(2.8)$ must be positive. Thus we have

$$
\begin{aligned}
\left(\sum_{i=1}^{n-1} a_{i}\right)^{2} & >(n-2)\left\{(n-2) \sum_{i=1}^{n-1} a_{i}^{2}-2 \sum_{i<j \leq n-1} a_{i} a_{j}+(n-1) k^{2}\right\} \\
& =(n-2)\left\{(n-2) \sum_{i=1}^{n-1} a_{i}^{2}-\left(\sum_{i=1}^{n-1} a_{i}\right)^{2}+\sum_{i=1}^{n-1} a_{i}^{2}+(n-1) k^{2}\right\} \\
& =(n-2)\left\{(n-1)\left(\sum_{i=1}^{n-1} a_{i}^{2}+k^{2}\right)-\left(\sum_{i=1}^{n-1} a_{i}\right)^{2}\right\},
\end{aligned}
$$

from which

$$
\sum_{i=1}^{n-1} a_{i}^{2}+k^{2}<\frac{1}{n-2}\left(\sum_{i=1}^{n-1} a_{i}\right)^{2}
$$

This inequality is the same type as (2.6). Continuing the same process as that which led to (2.9) from (2.6), we get at last $k^{2}<2 a_{1} a_{2}$.

Since for any $a_{i}, i=1, \ldots, n$, the same quadratic inequality as (2.8) must be satisfied, we can conclude that $(2.7)$ is valid. This completes the proof.

3. Pinching problem for the length of the second fundamental tensor. In this section we prove the following

Theorem 3.1. Let $M$ be a submanifold of dimension $n$ immersed in a manifold $\bar{M}$ of nonnegative constant curvature $c$ of dimension $(n+p)$. Suppose that the connection of the normal bundle of $M$ in $\bar{M}$ is flat and that the mean curvature vector field of $M$ is parallel with respect to the connection of the normal bundle. If the function $f=\Sigma_{A=1}^{p}$ trace $H_{A}^{2}$ is constant in $M$, and the inequality

$$
\sum_{A=1}^{p} \operatorname{trace} H_{A}^{2}<\frac{1}{n-1} \sum_{A=1}^{p}\left(\operatorname{trace} H_{A}\right)^{2}
$$


is satisfied, then $M$ is a totally umbilical submanifold.

Proof. From the assumption it follows that $M$ has no minimal point. So we can choose the first unit normal vector $N_{1}$ to $M$ in the direction of the mean curvature vector $N$. Then by the definition of the mean curvature vector we can easily see that

$$
\text { trace } H_{A}=0, \quad A=2,3, \cdots, p \text {, }
$$

and the formula (2.4) can be rewritten as

$$
\begin{aligned}
1 / 2 \Delta f= & c\left(n f-\left(\text { trace } H_{1}\right)^{2}\right)+\sum_{A=1}^{p} g\left(\nabla^{*} H_{A}, \nabla^{*} H_{A}\right) \\
& +\sum_{A=1}^{p}\left\{\left(\text { trace } H_{1}\right)\left(\text { trace } H_{1} H_{A}^{2}\right)-\left(\text { trace } H_{1} H_{A}\right)^{2}\right\} \\
& +\sum_{A=2}^{p} \sum_{B=1}^{p}\left\{\left(\text { trace } H_{A}\right)\left(\text { trace } H_{A} H_{B}^{2}\right)-\left(\text { trace } H_{A} H_{B}\right)^{2}\right\} \\
= & c\left(n f-\left(\text { trace } H_{1}\right)^{2}\right)+\sum_{A=1}^{p} g\left(\nabla^{*} H_{A}, \nabla^{*} H_{A}\right) \\
& +\left(\text { trace } H_{1}\right)\left(\text { trace } H_{1}^{3}\right)-\left(\text { trace } H_{1}^{2}\right)^{2} \\
& +\sum_{A=2}^{p}\left\{\left(\text { trace } H_{1}\right)\left(\text { trace } H_{1} H_{A}^{2}\right)-\left(\text { trace } H_{1} H_{A}\right)^{2}\right\} \\
& -\sum_{A=2}^{p}\left(\text { trace } H_{A} H_{1}\right)^{2}-\sum_{A=2}^{p} \sum_{B=2}^{p}\left(\text { trace } H_{A} H_{B}\right)^{2} .
\end{aligned}
$$

Since the connection of the normal bundle is flat the second fundamental tensors $H_{A}$ 's are simultaneously diagonalizable at each point of $M$, that is, there exist certain $n$ mutually orthogonal unit vectors $E_{1}, \ldots, E_{n}$ such that $H_{1} E_{i}=a_{i} E_{i}$ and $H_{A} E_{i}=\lambda_{i}^{A} E_{i}(A=2, \cdots, p)$. Then we have from (3.3)

$$
\begin{aligned}
1 / 2 \Delta f= & c\left(n f-\left(\sum_{i=1}^{n} a_{i}\right)^{2}\right)+\sum_{A=1}^{p} g\left(\nabla^{*} H_{A}, \nabla^{*} H_{A}\right) \\
& +\left(\sum_{i=1}^{n} a_{i}\right)\left(\sum_{i=1}^{n} a_{j}^{3}\right)-\left(\sum_{i=1}^{n} a_{i}^{2}\right)^{2} \\
& +\sum_{A=2}^{p}\left\{\left(\sum_{i=1}^{n} a_{i}\right)\left(\sum_{j=1}^{n} a_{j}\left(\lambda_{j}^{A}\right)^{2}\right)-\left(\sum_{i=1}^{n} a_{i} \lambda_{i}^{A}\right)^{2}\right\} \\
& -\sum_{A=2}^{p}\left(\sum_{i=1}^{n} \lambda_{i}^{A} a_{i}\right)^{2}-\sum_{A=2}^{p} \sum_{B=2}^{p}\left(\sum_{i=1}^{n} \lambda_{i}^{A} \lambda_{i}^{B}\right)^{2},
\end{aligned}
$$


from which, using Lemma 2.1 and $\sum_{i=1}^{n} \lambda_{i}^{A}=0(A=2, \cdots, p)$, we get

$$
\begin{aligned}
1 / 2 \Delta f= & c\left(n f-\left(\sum_{i=1}^{n} a_{i}\right)^{2}\right)+\sum_{A=1}^{p} g\left(\nabla^{*} H_{A}, \nabla^{*} H_{A}\right) \\
& +\sum_{i<j}\left(a_{i} a_{j}+\sum_{A=2}^{p} \lambda_{i}^{A} \lambda_{j}^{A}\right)\left(a_{i}-a_{j}\right)^{2} \\
& +\sum_{B=2}^{p} \sum_{i<j}\left(a_{i} a_{j}+\sum_{A=2}^{p} \lambda_{i}^{A} \lambda_{j}^{A}\right)\left(\lambda_{i}^{B}-\lambda_{j}^{B}\right)^{2} .
\end{aligned}
$$

Applying Lemma 2.2 to $a_{1}, \cdots, a_{n}$ and $\sum_{A=2}^{p} \sum_{i=1}^{n}\left(\lambda_{i}^{A}\right)^{2}$, we get

$$
2 a_{i} a_{j}>\sum_{A=2}^{p} \sum_{i=1}^{n}\left(\lambda_{i}^{A}\right)^{2} \geq \sum_{A=2}^{p}\left\{\left(\lambda_{i}^{A}\right)^{2}+\left(\lambda_{j}^{A}\right)^{2}\right\} \geq 2 \sum_{A=2}^{p}\left|\lambda_{i}^{A}\right|\left|\lambda_{j}^{A}\right|,
$$

for any pair of $i, j=1,2, \ldots, n$. Comparing (3.4) and (3.5), we know that each term of the right-hand members of (3.4) is not negative. So if $f$ is a constant, we have $a_{i}=a_{j}$ and $\lambda_{i}^{B}=\lambda_{j}^{B}$ for $B=2, \cdots, p$ and any pair of $i$ and $j$. This shows that $M$ is totally umbilical and completes the proof.

If the submanifold $M$ has constant scalar curvature, then by (2.2) and (1.3), it follows that $f$ is constant. Hence we have

Theorem 3.2. Let $M$ be an n-dimensional submanifold with constant scalar curvature which is immersed in a manifold of nonnegative constant curvature of dimension $(n+p)$. Suppose that the connection of the normal bundle of $M$ is flat and that the mean curvature vector field of $M$ is parallel with respect to the connection of the normal bundle. If the inequality (3.1) is satisfied, then $M$ is a totally umbilical submanifold.

Let $M$ be a compact submanifold of $\bar{M}$. Then by the well-known Bochner's lemma (for example [5]), it follows that $f$ is constant, because of the fact that the right-hand members of (3.4) are nonnegative. Hence we have

Theorem 3.3. Let $M$ be a compact submanifold of dimension $n$ immersed in a manifold $\bar{M}$ of nonnegative constant curvature of $(n+p)$-dimension and suppose that the connection of the normal bundle is flat. If the mean curvature vector field is parallel with respect to the connection of the normal bundle and the inequality (3.1) is satisfied, then $M$ is a totally umbilical submanifold.

Remark 1. Using (1.3), we can rewrite (3.1) as

$$
K>n(n-1) c+(n-2) \sum_{A=1}^{p} \operatorname{trace} H_{A}^{2} \text {. }
$$

From (3.6), we know that Theorem 3.3 is a generalization of the classical theorem of H. Liebmann [2], because, if $n=2, p=1$ and the ambient manifold is Euclidean 3-space, (3.6) shows that the Gauss curvature is positive. 
Remark 2. We consider $S^{n-1} \times E^{1}$ which is standardly immersed in $(n+p)$ dimensional Euclidean space. Then $S^{n-1} \times E^{1}$ has constant scalar curvature and parallel mean curvature vector field with respect to the connection of the normal bundle. Moreover, the connection of the normal bundle of $S^{n-1} \times E^{1}$ in $E^{n+p}$ is flat. But $S^{n-1} \times E^{1}$ satisfies the equality

$$
\sum_{A=1}^{p} \text { trace } H_{A}^{2}=\frac{1}{n-1} \sum_{A=1}^{p}\left(\text { trace } H_{A}\right)^{2} .
$$

Thus, the estimation (3.1) in Theorem 3.3 is the best possible, when the ambient manifold is a Euclidean space.

On the other hand, for a totally umbilical submanifold of any Riemannian manifold we have from (1.7)

$$
\sum_{A=1}^{p} \operatorname{trace} H_{A}^{2}=\frac{1}{n} \sum_{A=1}^{p}\left(\operatorname{trace} H_{A}\right)^{2} \text {. }
$$

This shows that if $M$ is totally umbilical and not minimal, then the condition (3.1) holds.

\section{BIBLIOGRAPHY}

1. J. A. Erbacher, Isometric immersions of Riemannian manifolds into space forms, Thesis, Brown University, Providence, R. I., 1970.

2. H. Liebmann, Über die Verbiegung der geschlassenen Flächen positiver Krummung, Math. Ann. 53 (1900), 91-112.

3. K. Nomizu and B. Smyth, A formula of Simons' type and hypersurfaces with constant mean curvature, Differential Geometry 3 (1969), 367-377. MR $42 \# 1018$.

4. M. Okumura, Hypersurfaces and a pinching problem on the second fundamental tensor (to appear).

5. K. Yano and S. Bochner, Curvature and Betti numbers, Ann. of Math. Studies, no. 32, Princeton Univ. Press, Princeton, N. J., 1953. MR 15, 989.

6. K. Yano and S. Ishihara, Submanifolds with parallel mean curvature vector, J. Differential Geometry 6 (1971), 95-118.

DEPARTMENT OF MATHEMATICS, MICHIGAN STATE UNIVERSITY, EAST LANSING, MICHIGAN 48823

Current address: Deparment of Mathematics, Saitama Uni versity, Urawa, Japan 